\title{
HPLC-UV Method Development and Validation of Potato Sprout Inhibitor 1,4-Dimethylnaphthalene Using Different Systems
}

\author{
Nidhal S. Mohammed, ${ }^{1}$ T. H. Flowers, ${ }^{2}$ and H. J. Duncan ${ }^{2}$ \\ ${ }^{1}$ School of Chemistry, University of Zakho, Zakho, Kurdistan Region, Iraq \\ ${ }^{2}$ School of Chemistry, University of Glasgow, Joseph Black Building, Glasgow G12 8QQ, UK \\ Correspondence should be addressed to Nidhal S. Mohammed; nidhalsher@yahoo.com
}

Received 25 July 2014; Accepted 8 September 2014; Published 16 September 2014

Academic Editor: Joselito P. Quirino

Copyright ( 2014 Nidhal S. Mohammed et al. This is an open access article distributed under the Creative Commons Attribution License, which permits unrestricted use, distribution, and reproduction in any medium, provided the original work is properly cited.

1,4-Dimethylnaphthalene (1,4-DMN) is effective sprout suppressant used in potato stores in many countries in the world. High residue levels of this compound on the potatoes and in other environmental samples are considered for human health and environmental risks. Determination of the residue requires specific analytical methods to be developed and validated. In this study, HPLC-UV was selected for validating a separation method based on reversed phase for the analysis of 1,4-DMN using 2-methylnaphthalene (2-MeN) as internal standard testing three HPLC systems. Under the same chromatographic conditions, all three systems achieved good separation on a Jones column (Hypersil ODS $5 \mu \mathrm{m}, 250 \mathrm{~mm} \times 4.6 \mathrm{~mm}$ ) at ambient temperature isocratically using $70 \%$ acetonitrile as mobile phase at a flow rate of $1.5 \mathrm{~mL} \mathrm{~min}^{-1}, 20 \mu \mathrm{L}$ injection volume, a run time of $10 \mathrm{~min}$, and a detection wavelength of $228 \mathrm{~nm}$. All three systems showed high precision, good linearity, and low limit of detection (LOD) and quantification (LOQ); particularly, the SpectraSERIES UV100-autosampler system offered lower values of LOD (0.001$\left.0.004 \mu \mathrm{g} \mathrm{mL}^{-1}\right)$ and LOQ $\left(0.002-0.013 \mu \mathrm{g} \mathrm{mL}^{-1}\right)$ for both compounds. This system can be used for the quantitative determination of $1,4-\mathrm{DMN}$ residue in potato and environmental samples.

\section{Introduction}

1,4-Dimethylnaphthalene (1,4-DMN) is an effective chemical used for sprout control and quality management and known by the trade name DMN; it is available commercially mainly as 1,4SIGHT in USA, Canada, and New Zealand. However, the commercial use of $1,4-\mathrm{DMN}$ as a naturally occurring potato sprout inhibitor $[1,2]$ is claiming investigation of the effective amount required for successful long-term storage and to understand the possible risks to the environment regarding waste peel, water, and soil. Therefore, validated analytical methods are required. The scientific literature for the determination of this polyaromatic hydrocarbon of 1,4DMN is very limited. Few studies have been reported to analyse the residue of 1,4-DMN in potato samples using GC [3-5]. Nowadays, the increasing availability of HPLC analysis proved to have many advantages for pesticide residue analysis in food and environmental samples compared to GC analysis particularly polyaromatic hydrocarbons mainly owing to compounds stability, high speed, and producing reproducible response with various kinds of solvents and columns analysing large volume of samples $(\sim 500 \mu \mathrm{L})[6,7]$. The only study which has been found regarding the analysis of 1,4-DMN by HPLC-UV is separation method based on reversed phase (RP) through studying the behaviour of a mixture of seven isomers of 1,4-dimethylnaphthalene and other related compounds under different chromatographic conditions [8]. The best separation was achieved using a Supelco Supelcosil ODS2 $\left(\mathrm{C}_{18}\right)$ column at a temperature of $12^{\circ} \mathrm{C}$ with $40 \%(\mathrm{v} / \mathrm{v})$ acetonitrile as a mobile phase at a flow rate of $1.5 \mathrm{~mL} \mathrm{~min}^{-1}$. The method provided a good separation for most components in the mixture; in addition, good linearity and precision were obtained through method validation; however, long chromatographic run time of $75 \mathrm{~min}$ 
was required. A short run time is usually required to analyse more samples on a daily basis.

The work reported in this study describes the development of isocratic HPLC-UV method for the analysis of the potato sprout inhibitor 1,4-DMN and its internal standard 2-MeN employing three HPLC systems including a manual injection valve or autosampler. A further objective was to compare the sensitivity of a Hitachi diode array detector (DAD) and a SpectraSERIES UV100 single wavelength detector for the determination of these compounds. Validating the HPLC method is crucial to prove the acceptability of the method and suitability for its intended purpose. RPHPLC method was validated for four major parameters including repeatability, linearity, the limit of detection (LOD), and the limit of quantification (LOQ). This initial step was important to select the best HPLC system optimising the chromatographic conditions prior to determination of 1,4DMN in potato and environmental samples.

\section{Materials and Methods}

2.1. Reagents. Analytical reagents were used from 1,4-dimethylnaphthalene (95\%) and 2-methylnaphthalene (97\%), which were purchased from Sigma-Aldrich Chemi $\mathrm{GmbH}$ (Germany). Stock solutions of $10000 \mu \mathrm{g} \mathrm{mL}^{-1}$ of each were prepared by dissolving $1 \mathrm{~g}$ in $100 \mathrm{~mL}$ of acetonitrile (HPLC grade, Fisher, UK). These individual stock standard solutions were stored in a refrigerator and used to prepare the working solutions at different concentrations. The standard solutions were warmed to room temperature prior to injection.

2.2. Instrumentation. Three HPLC systems were used during this work; the brief details of these systems are summarised below.

(i) Hitachi (autosampler) system: an autosampler Merck Hitachi L-7200 and Merck Hitachi L-7100 pump were coupled to a Merck Hitachi L-4500 diode array detector (DAD); the output was recorded by Merck Hitachi L-7000 software version 4.1.

(ii) SpectraSERIES UV100 (manual) system: the manual injector was a Rheodyne model 7125, and the pump used was a Cecil 1100 Series; these were connected with the thermoseparation products SpectraSERIES UV100 detector and Dionex peaknet software.

(iii) SpectraSERIES UV100 (autosampler) system: an autosampler Merck Hitachi L-7200 and Merck Hitachi L-7100 pump were coupled to thermo-separation products SpectraSERIES UV100 detector and Dionex peaknet software.

2.3. Chromatographic Conditions. Separation was performed on the three HPLC systems under the same conditions using a Jones chromatography column (Hypersil ODS $5 \mu \mathrm{m}, 250 \mathrm{~mm}$ $\times 4.6 \mathrm{~mm}$ I.D.) at ambient temperature. The mobile phase consisted of $70 \%$ acetonitrile and $30 \%$ water at a flow rate of $1.5 \mathrm{~mL} \mathrm{~min}^{-1}$ and a run time of $10 \mathrm{~min}$. The injection volume of the sample was $20 \mu \mathrm{L}$ and the detection was set at a wavelength of $228 \mathrm{~nm}$.
2.4. Preparation the Mobile Phase. The mobile phase for HPLC analysis was prepared from acetonitrile and water $(\mathrm{v} / \mathrm{v})$. The water used for preparation of the mobile phase was supplied from an Elga Purelab Option deionizer model LA613 and then filtered through a Supor-200 membrane filter ( $47 \mathrm{~mm} 0.2 \mu \mathrm{m}$ ). The mobile phase was degassed using helium gas. Stabilising the temperature was controlled by insulating the mobile phase by placing the reservoir of the mobile phase in a polystyrene box. In addition, the mobile phase was prepared the day before it was required for analysis and kept overnight at a fixed room temperature of $20^{\circ} \mathrm{C}$.

2.5. Assessment of the Precision for HPLC Systems. The precision of the three HPLC systems was evaluated following repeated injections $(n=10)$ of $1 \mu \mathrm{g} \mathrm{mL}^{-1}$ mixture of 1,4 -DMN and $2-\mathrm{MeN}$ by calculating the relative standard deviation (RSD\%) through the peak area as follows:

$$
\mathrm{RSD} \%=\left(\frac{\mathrm{SD}}{\mathrm{M}}\right) * 100,
$$

where SD is the standard deviation of the peak area and M is the mean of the peak area.

2.6. Linearity. Two sets of mixed 1,4-DMN and $2-\mathrm{MeN}$ standards were prepared. The first set of standards consisted of the following concentrations of $0.2,0.4,0.6,0.8$, and $1.0 \mu \mathrm{g} \mathrm{mL}^{-1}$. The second set of standards consisted of lower concentrations than the first set of $0.02,0.04,0.06,0.08$, and $0.10 \mu \mathrm{g} \mathrm{mL}^{-1}$. The different sets of standards were injected as duplicates into the three HPLC systems. Linearity was evaluated according to the relationship between the peak area of the compound and its concentration. Excel software was employed to plot the calibration curve for each compound in the solution. From the regression line, the coefficient of determination $\left(R^{2}\right)$ was obtained to statistically assess the linear relationship.

2.7. Limit of Detection and Quantification. The limit of detection (LOD) and the limit of quantification (LOQ) of 1,4-DMN and its internal standard $(2-\mathrm{MeN})$ in solution were calculated by two approaches; the first approach was by ten replicate injections of a single solution of $0.01 \mu \mathrm{g} \mathrm{mL}^{-1}$ (just above the expected LOQ, approximately $5 *$ LOQ) into the three HPLC systems as follows:

$$
\begin{aligned}
& \text { peak area for } \mathrm{LOD}=3 * \mathrm{SD}, \\
& \mathrm{LOD}=\text { Peak area for } \mathrm{LOD} *(\text { Conc. } / \mathrm{M}), \\
& \text { peak area for } \mathrm{LOQ}=10 * \mathrm{SD}, \\
& \mathrm{LOQ}=\text { Peak area for } \mathrm{LOQ} *(\text { Conc. } / \mathrm{M}),
\end{aligned}
$$

where SD and M are the standard deviation and mean of the peak area $(n=10)$, respectively, and Conc. is the concentration of the solution injected.

The second approach to determine the LOD and the LOQ was based upon the statistical data from plotting the calibration curve in the Microsoft Excel sheet at the lowest range of the concentrations $\left(0.02-0.1 \mu \mathrm{g} \mathrm{mL}^{-1}\right)$. These statistical data consist of the standard deviation (SD) and the 


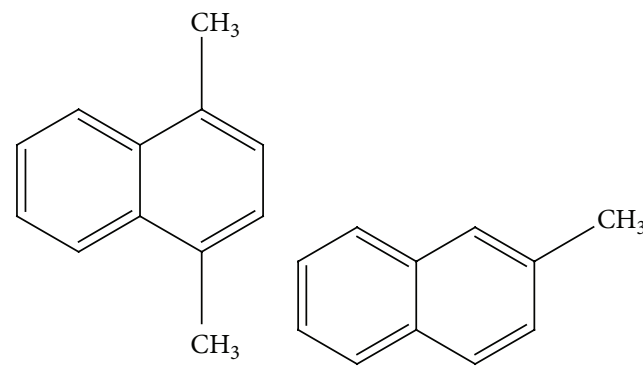

(a)

(b)

FIGURE 1: The chemical structure of (a) 1,4-dimethylnaphthalene and (b) 2-methylnaphthalene.

TABLE 1: RSD\% values of the peak area of 1,4-DMN and 2-MeN on three HPLC systems.

\begin{tabular}{lccc}
\hline HPLC system & Injector & RSD\% of peak areas $(n=10)$ & $1,4-\mathrm{DMN}$ \\
\hline Hitachi DAD & & $2-\mathrm{MeN}$ & 0.11 \\
SpectraSERIES UV100 & Autosampler & 0.07 & 0.82 \\
SpectraSERIES UV100 & Manual & 0.91 & 0.16 \\
\hline
\end{tabular}

slope of the regression line. The LOD and the LOQ were determined according to the following equations:

$$
\begin{aligned}
& \mathrm{LOD}=3\left(\frac{\mathrm{SD}}{\text { slope }}\right), \\
& \mathrm{LOQ}=10\left(\frac{\mathrm{SD}}{\text { slope }}\right) .
\end{aligned}
$$

\section{Results and Discussion}

3.1. Chromatographic Conditions. A reversed phase-HPLC technique was selected for the determination of 1,4dimethylnaphthalene (1,4-DMN) and 2-methylnaphthalene $(2-\mathrm{MeN})$ as an appropriate internal standard to mimic the variation of any loss of $1,4-\mathrm{DMN}$ during the sample preparation or instrumental analysis. 2-MeN was selected from the different isomers and related compounds due to its structural similarity to $1,4-\mathrm{DMN}$ as shown in Figure 1 and its good resolution from $1,4-\mathrm{DMN}$ in a mixed standard solution compared with other compounds. Moreover, the solubility of $2-\mathrm{MeN}$ in water is higher than other related naphthalene compounds, which is important when investigating 1,4-DMN in water samples.

To develop a simple method achieving good separation of 1,4-DMN and its internal standard 2-MeN, three HPLC systems were employed under the same chromatographic conditions. The three HPLC systems were used to compare two aspects, including the sample injection method and the detector sensitivity. Sample injection can be done either manually using a manual injection valve or automatically by an autosampler. The choice of the detector is one of the main considerations that should be taken into account when developing an HPLC analytical method which principally depends on the limit of detection required for the target analyte.
The chromatographic conditions chosen in this study achieved good separation with high resolution between the closely eluted 1,4-DMN peak and $2-\mathrm{MeN}$ peak at a short retention time, approximately between 5.5-7.0 and 4.5$5.5 \mathrm{~min}$, respectively, as shown in Figure 2. However, the slight variability of the retention time between chromatographic systems can be due to various factors regarding the different specification of each HPLC system and column temperature which was ambient and not controlled.

3.2. Assessment of the Precision for the HPLC Systems. Precision is important to achieve consistent quantitative data. A peak area was preferred for precision calculation over peak height due to the variability of using the peak height, which may be effected by some parameters such as the column temperature, mobile phase composition, pump pressure, and volume injection. It was stated that if the flow control is good but solvent composition cannot be maintained precisely, peak area measurement is better because area is relatively independent of composition [9].

To achieve the required precision measurement, the ambient temperature of the apparatus room should be controlled and maintained [10]. In the present study, the major focus was to improve the precision of the peak area by stabilising the column temperature. Some temperature variation of the mobile phase and standard solution had an effect on the column temperature. Therefore, the temperature of the chromatographic system needs to be fixed at (or slightly above) ambient temperature, which is commonly between $20^{\circ} \mathrm{C}$ and $25^{\circ} \mathrm{C}$. Furthermore, in order to overcome the temperature effect on the retention time and precision measurement, controlling the column temperature is required using a column oven coupled with cooling devices to obtain stable chromatographic conditions. A big improvement in the precision was achieved after stabilising the temperature as shown in Table 1. 


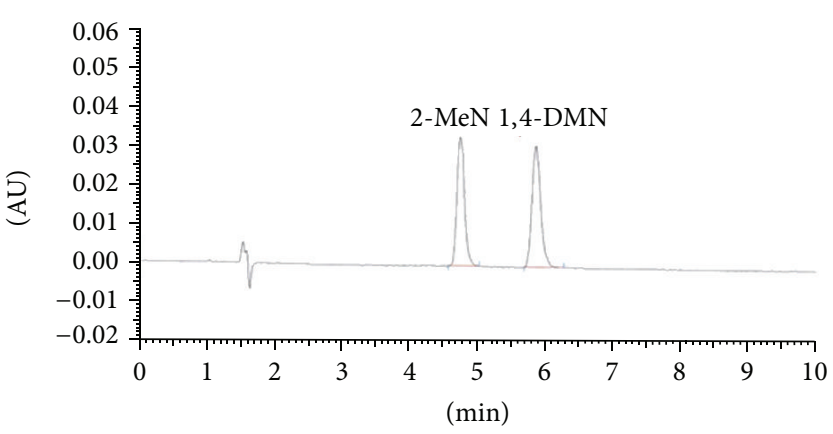

(a)

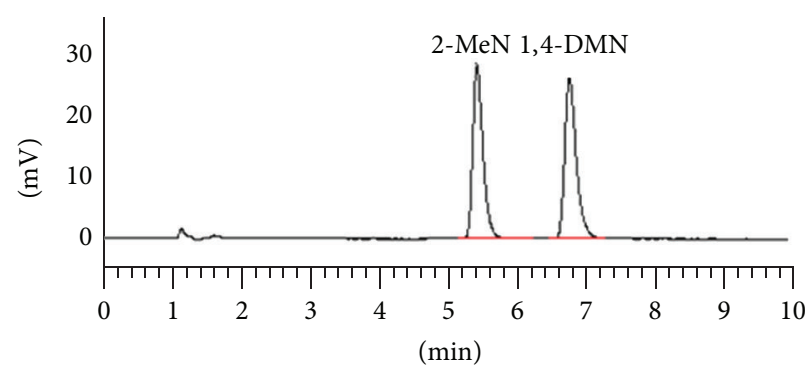

(b)

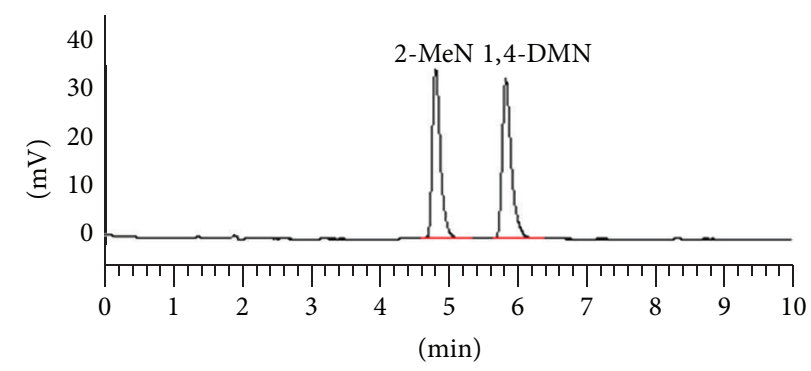

(c)

Figure 2: Chromatograms of $1 \mu \mathrm{g} \mathrm{mL}^{-1}$ mixture of 1,4-DMN and 2-MeN of three HPLC systems: (a) Hitachi DAD-autosampler, (b) SpectraSERIES UV100-manual injector, and (c) SpectraSERIES UV100-autosampler.

TABLE 2: Coefficients of determination $\left(R^{2}\right)$ values of the calibration curve for studied compounds at two ranges in concentration injected onto three HPLC systems.

\begin{tabular}{|c|c|c|c|c|}
\hline \multirow{2}{*}{ HPLC system } & \multirow{2}{*}{ Injector } & \multirow{2}{*}{ Conc. range $\left(\mu \mathrm{g} \mathrm{mL}^{-1}\right)$} & \multicolumn{2}{|c|}{$R^{2}$} \\
\hline & & & 2-MeN & 1,4-DMN \\
\hline \multirow{2}{*}{ Hitachi DAD } & \multirow{2}{*}{ Autosampler } & $0.02-0.1$ & 0.980 & 0.980 \\
\hline & & $0.2-1.0$ & 0.999 & 0.999 \\
\hline \multirow{2}{*}{ SpectraSERIES UV100 } & \multirow{2}{*}{ Manual } & $0.02-0.1$ & 0.996 & 0.995 \\
\hline & & $0.2-1.0$ & 0.999 & 0.998 \\
\hline \multirow{2}{*}{ SpectraSERIES UV100 } & \multirow{2}{*}{ Autosampler } & $0.02-0.1$ & 0.999 & 0.998 \\
\hline & & $0.2-1.0$ & 0.999 & 0.999 \\
\hline
\end{tabular}

The three HPLC systems achieved good precision for both compounds (2-MeN and 1,4-DMN) as shown by RSD\% values of the peak area of less than $1 \%$. The value of RSD\% is suggested to be $\leq 1 \%$ as an appropriate precision criterion for repetitive injections to assess the precision of the instrument in analytical method validation [11, 12]. In addition, an RSD $\%$ of $1 \%$ or less is acceptable for the precision criteria in particular of the autosampler performance for at least six replicates [13].

In this study, the low RSD\% values indicated satisfactory repeatability of the HPLC method. However, the precision of the analytical method was slightly better on the autosampler injector systems (Hitachi DAD and SpectraSERIES UV100 systems) than SpectraSERIES UV100manual injector. Autosampler injection is more frequently used in standard HPLC equipment as it provides better reproducibility than manual injection.
3.3. Linearity of Calibration Curve. Assessment of the linearity of the calibration curve is recommended to prove the acceptability of any analytical method [11]. The linearity can be examined through the correlation coefficient $(r)$ which is often used as linearity measure of the calibration curve. In chemical correlation analysis, $R^{2}$ is the more exact term used [14]. In this study, the coefficient of determination $\left(R^{2}\right)$ of the regression line is suggested to be 0.990 or more. On this basis, the results illustrate a good linearity between the peak area and the concentrations of the standard solutions of each of 1,4-DMN and 2-MeN on all three HPLC systems as shown in the Table 2 .

3.4. Limit of Detection and Quantification. The LOD and LOQ were calculated using two approaches for the three HPLC systems as shown in Tables 3 and 4. 
TABLE 3: LOD and LOQ values for repeatability injection of $0.01 \mu \mathrm{g} \mathrm{mL} L^{-1}$ mixture of 2-MeN and 1,4-DMN on each HPLC system.

\begin{tabular}{|c|c|c|c|c|c|}
\hline \multirow{2}{*}{ HPLC system } & \multirow{2}{*}{ Injector } & \multicolumn{2}{|c|}{$\mathrm{LOD}\left(\mu \mathrm{g} \mathrm{mL}^{-1}\right)$} & \multicolumn{2}{|c|}{ LOQ $\left(\mu \mathrm{g} \mathrm{mL}^{-1}\right)$} \\
\hline & & $2-\mathrm{MeN}$ & $1,4-\mathrm{DMN}$ & 2-MeN & 1,4-DMN \\
\hline Hitachi DAD & Autosampler & 0.003 & 0.004 & 0.010 & 0.015 \\
\hline SpectraSERIES UV100 & Manual & 0.001 & 0.001 & 0.002 & 0.004 \\
\hline SpectraSERIES UV100 & Autosampler & 0.001 & 0.001 & 0.002 & 0.002 \\
\hline
\end{tabular}

TABLE 4: LOD and LOQ values on each HPLC system based on the statistical data for the calibration curve in the range $0.02-0.1 \mu \mathrm{g} \mathrm{mL}^{-1}$ for 2-MeN and 1,4-DMN.

\begin{tabular}{|c|c|c|c|c|c|}
\hline \multirow{2}{*}{ HPLC system } & \multirow{2}{*}{ Injector } & \multicolumn{2}{|c|}{$\operatorname{LOD}\left(\mu \mathrm{g} \mathrm{mL}^{-1}\right)$} & \multicolumn{2}{|c|}{ LOQ $\left(\mu \mathrm{g} \mathrm{mL}^{-1}\right)$} \\
\hline & & 2-MeN & $1,4-\mathrm{DMN}$ & 2-MeN & $1,4-\mathrm{DMN}$ \\
\hline Hitachi DAD & Autosampler & 0.014 & 0.017 & 0.046 & 0.057 \\
\hline SpectraSERIES UV100 & Manual & 0.007 & 0.008 & 0.024 & 0.028 \\
\hline SpectraSERIES UV100 & Autosampler & 0.004 & 0.004 & 0.013 & 0.013 \\
\hline
\end{tabular}

To compare the reliability of the presented results of LOD and LOQ for the two approaches, these values undoubtedly are different because the calculation of a standard deviation for each approach is different. The values for the repeated injections approach are lower and more realistic in practical use, whereas the graphical method shows higher values as the assumption of a constant standard deviation at all concentrations is probably not true. All three systems offered acceptable LOD and LOQ values despite the difference in the specification of the Hitachi DAD and SpectraSERIES UV100 detectors. DAD-autosampler HPLC system presented LOD and LOQ values for 1,4-DMN higher than SpectraSERIES UV100 detector-autosampler system. However, the diode array detector can be used to monitor multiwavelength and peak purity providing entire spectra of all the peaks during the chromatogram run [15].

\section{Conclusion}

Three HPLC systems were operated with the aim of validating analytical method for the determination of 1,4-dimethylnaphthalene and its internal standard 2methylnaphthalene choosing the best system. Excellent separation was achieved using the same chromatographic conditions with all three HPLC systems but using an autosampler coupled with a single wavelength detector (SpectraSERIES UV100) system gave the most precise results and good linearity of the calibration curves with lower limits of detection and quantification for 1,4-DMN. However, some essential modifications were required to achieve the best chromatographic conditions on this system such as the column oven and cooling device to overcome any temperature effects. This system can be used for the determination of 1,4-DMN in laboratory for the analysis of potato and environmental samples.

\section{Conflict of Interests}

The authors declare that there is no conflict of interests regarding the publication of this paper.

\section{Acknowledgment}

Nidhal S. Mohammed would like to thank the Iraqi government for financially supporting her Ph.D. scholarship.

\section{References}

[1] M. A. Campbell, A. Gleichsner, R. Alsbury, D. Horvath, and J. Suttle, "The sprout inhibitors chlorpropham and 1,4dimethylnaphthalene elicit different transcriptional profiles and do not suppress growth through a prolongation of the dormant state," Plant Molecular Biology, vol. 73, no. 1-2, pp. 181-189, 2010.

[2] J. L. Beveridge, J. Dalziel, and H. J. Duncan, “The assessment of some volatile organic compounds as sprout suppressants for ware and seed potatoes," Potato Research, vol. 24, no. 1, pp. 6176, 1981.

[3] N. R. Knowles, L. O. Knowles, and M. M. Haines, "1,4dimethylnaphthalene treatment of seed potatoes affects tuber size distribution," The American Journal of Potato Research, vol. 82, no. 3, pp. 179-190, 2005.

[4] T. D. Boylston, J. R. Powers, K. M. Weller, and J. Yang, "Comparison of sensory differences of stored Russet Burbank potatoes treated with CIPC and alternative sprout inhibitors," American Journal of Potato Research, vol. 78, no. 2, pp. 99-107, 2001.

[5] J. W. de Weerd, M. K. Thornton, and B. Shafii, "Sprout suppressing residue levels of 1,4 dimethylnaphthalene $(1,4 \mathrm{DMN})$ in potato cultivars," The American Journal of Potato Research, vol. 87, no. 5, pp. 434-445, 2010.

[6] J. F. Lawrence and R. Leduc, "Direct analysis of carbofuran and two nonconjugated metabolites in crops by high-pressure liquid chromatography with UV absorption detection," Journal of Agricultural and Food Chemistry, vol. 25, no. 6, pp. 1362-1365, 1977.

[7] J. F. Lawrence, "Advantages and limitations of HPLC in environmental analysis," Chromatographia, vol. 24, no. 1, pp. 45-50, 1987.

[8] M. D. Y. Oteef, Analysis of the potato sprout inhibitor 1, 4dimethylnaphthalene: HPLC method development and application [Ph.D. thesis], University of Glasgow, Glasgow, Scotland, 2008. 
[9] S. R. Bakalyar and R. A. Henry, "Variables affecting precision and accuracy in high-performance liquid chromatography," Journal of Chromatography A, vol. 126, no. 1, pp. 327-345, 1976.

[10] R. P. W. Scott and C. E. Reese, "Precision of contemporary liquid chromatographic measurements," Journal of Chromatography A, vol. 138, no. 2, pp. 283-307, 1977.

[11] J. M. Green, "A practical guide to analytical method validation," Analytical Chemistry, vol. 68, no. 9, pp. 305-309, 1996.

[12] G. A. Shabir, W. John Lough, S. A. Arain, and T. K. Bradshaw, "Evaluation and application of best practice in analytical method validation," Journal of Liquid Chromatography and Related Technologies, vol. 30, no. 3, pp. 311-333, 2007.

[13] G. Hall and J. W. Dolan, "Performance qualification of LC systems," LC-GC North America, vol. 20, no. 9, pp. 842-848, 2002.

[14] O. Exner and K. Zvára, "Coefficient of determination in some atypical situations: use in chemical correlation analysis," Journal of Physical Organic Chemistry, vol. 12, no. 2, pp. 151-156, 1999.

[15] V. T. Remcho, H. M. McNair, and H. T. Rasmussen, "HPLC method development with the photodiode array detector: a laboratory experiment," Journal of Chemical Education, vol. 69, no. 4, pp. 117-119, 1992. 

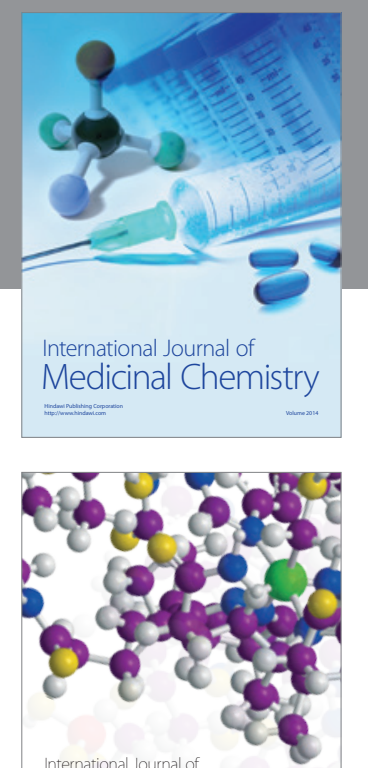

\section{Carbohydrate} Chemistry

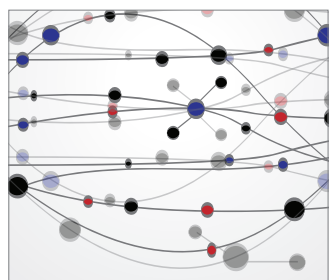

The Scientific World Journal
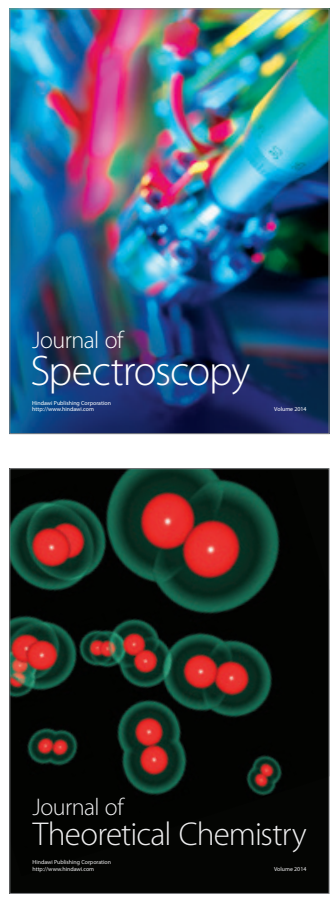
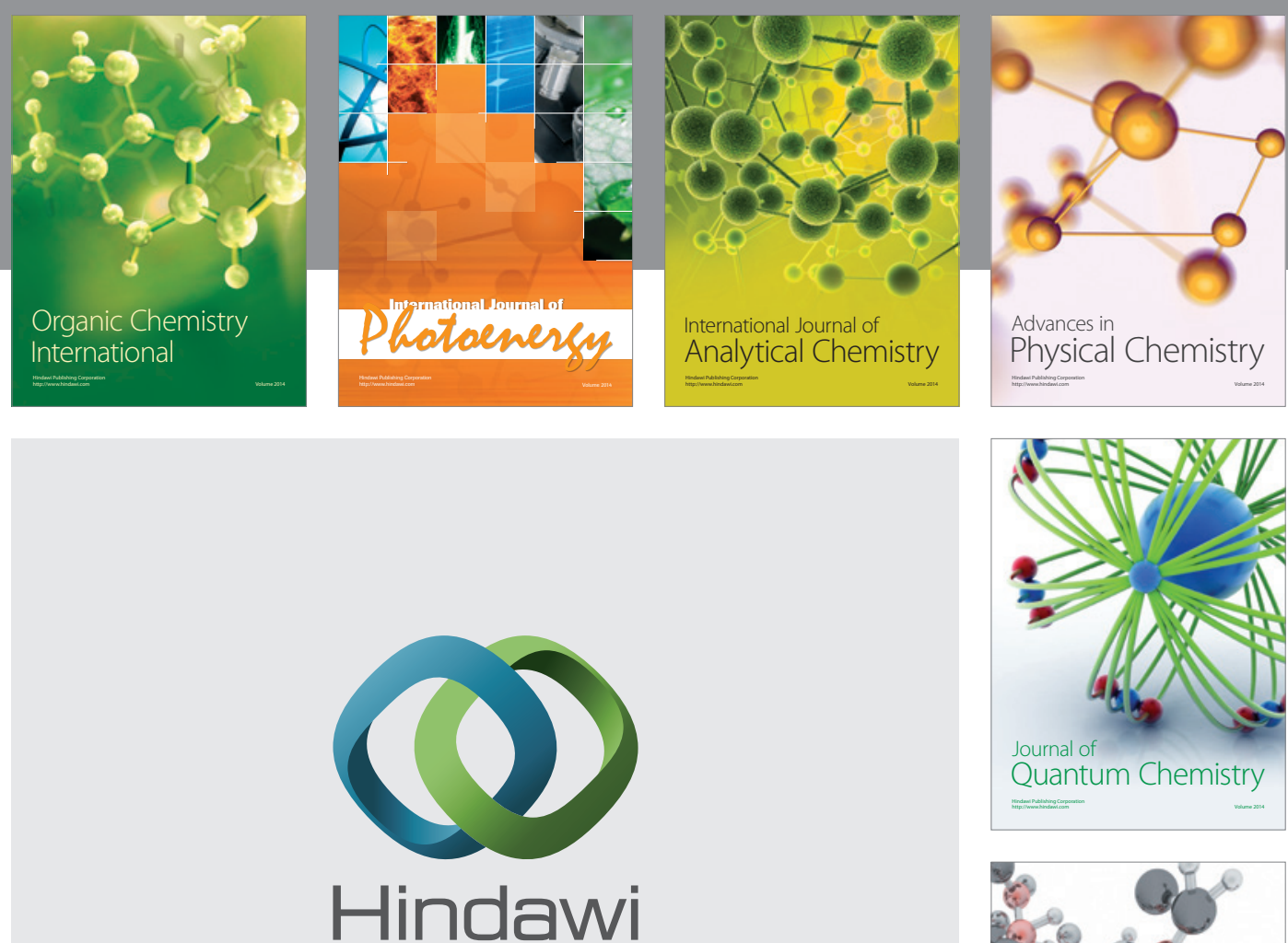

Submit your manuscripts at

http://www.hindawi.com

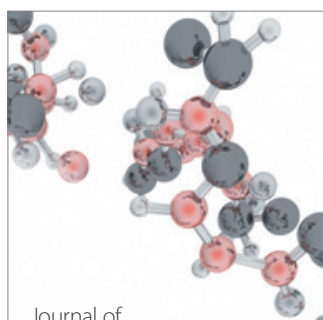

Analytical Methods

in Chemistry

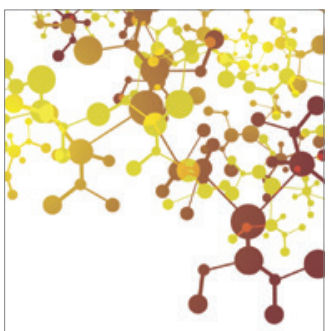

Journal of

Applied Chemistry

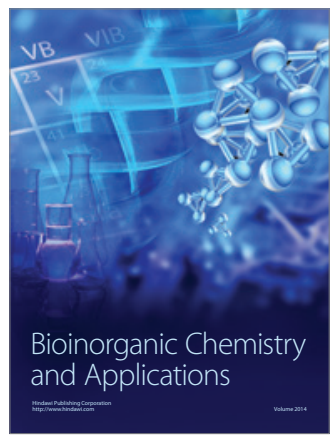

Inorganic Chemistry
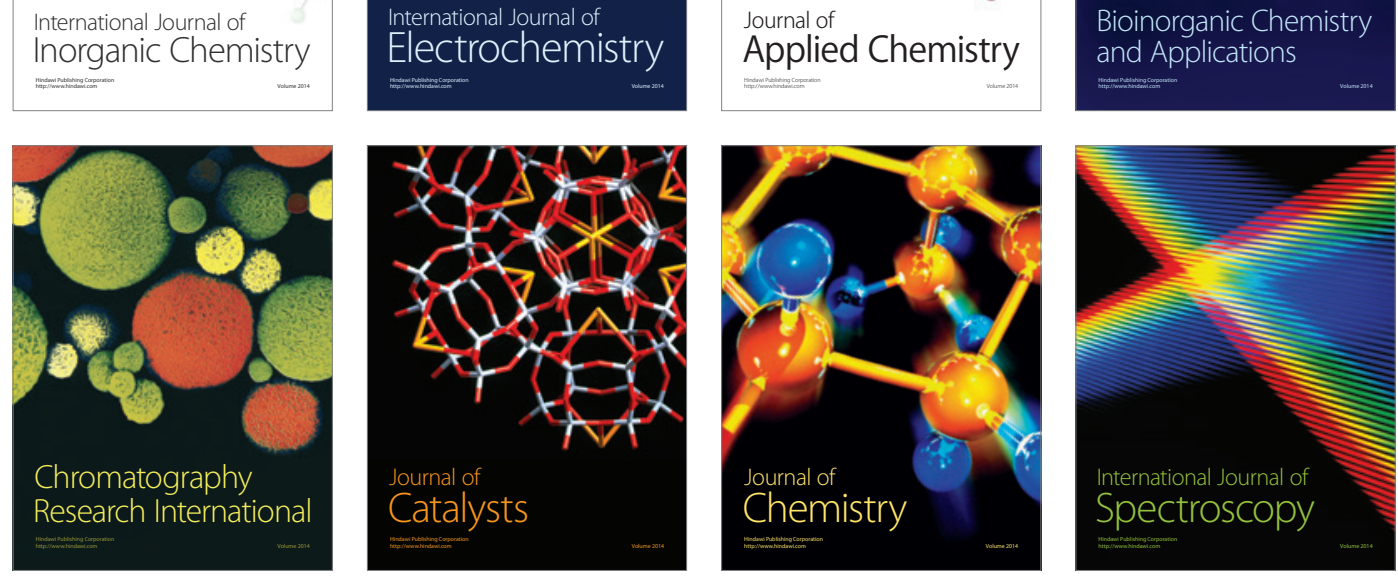